\title{
Effect of Hall Current on Hydromagnetic Boundary Layer in Rotating Dusty Fluid with Exponential Pressure Gradient
}

\author{
B. J. Gireesha ${ }^{1}$, S. Manjunatha ${ }^{2}$, H. J. Lokesh ${ }^{3}$, and C. S. Bagewadi ${ }^{4}$ \\ ${ }^{1,2,3,4}$ Department of Studies and Research in Mathematics, Kuvempu University, Shankaraghatta-577 451, \\ Shimoga, Karnataka, INDIA.
}

\begin{abstract}
An asymptotic analysis of an unsteady hydromagnetic boundary layer flow of an incompressible viscous conducting fluid with uniform distribution of dust particle bounded by semi-infinite plate in the presence of exponential decaying pressure gradient is investigated. The exact solutions of the boundary layer equations are obtained by asymptotic behaviour of Laplace transform treatment. The structure of velocity distribution is investigated and the effect of various physical parameters likes magnetic parameter, Ekman parameter and Hall current parameter on velocities of both fluid and dust phase are depicted graphically.
\end{abstract}

Key Words: Dusty fluid, pressure gradient, velocity of dust phase and fluid phase, rotating dusty fluid, Ekman number, Hall parameter.

\section{Introduction}

A number of industrially important fluids such as molten plastics, polymers, pulps and foods exhibit non-Newtonian fluid behavior (Nakayama [9]). Due to the growing use of these non-Newtonian materials in various manufacturing and processing industries, considerable efforts have been directed towards understanding their flow characteristics. The magnetohydrodynamic under the influence of viscous forces taking Hall current into an account is important in MHD power generation, cooling of nuclear reactors and in several astrophysical situations. The theory of rotating fluids is highly important due to its occurrence in various natural phenomena and for its applications in various technological situations which are directly governed by the action of Coriolis force. The broad subjects of Oceanography, Meteorology, Atmospheric science and Limnology all contain some important and essential features of rotating fluids.

The fluid flow problems in rotating medium have attracted many scholars and there appeared a number of studies in literature viz. Tiwari and Kamal Singh [1] have obtained solution for an asymptotic analysis of an unsteady hydromagnetic boundary layer flow generated impulsively in compressible viscous conducting fluid with uniform distribution of dust particle bounded by semi-infinite plate. Prasada Rao and Krishna [2] studied the Hall Effect on unsteady hydromagnetic flow. Debnath. [3] has discussed effect of hall current on unsteady hydromagnetic flow past a porous plate in a rotating fluid system. Kanch and Jana [5] have studied Hall effects on unsteady hydromagnetic flow past a rotating disk are investigated when the fluid at infinity rotates about non-coincident axes. Saffman [6] has initiated and investigated the effect of dusty particles on the stability of the laminar flow of an incompressible fluid with constant mass concentration of dust particles. Michael and Miller [11] have discussed the motion of dusty gas occupying the semi infinite space above a rigid plane boundary. Ghosh, Anwar Beg and Zueco [12] have studied the hydromagnetic natural convection boundary layer flow past an infinite vertical flat plate under the influence of a transverse magnetic field with magnetic induction effects.

The present paper aims at studying the effect of Hall current on hydromagnetic flow on an oscillating plate in a rotating dusty fluid under varying time dependent pressure gradient. Laplace Transform technique is employed to obtain the solution. But its exact inversion would be extremely difficult, so the asymptotic behavior of the solution has been analyzed for both small and large time to highlight the transient approach to the steady flow and other physical process involved in it. For the obtained solution the effect of physical parameter like magnetic parameter, Ekman parameter and Hall current parameter are studied.

\section{Mathematical Formulation}

Consider an unsteady flow induced in a semi-infinite plate of an electrically conducting incompressible viscous fluid with uniform distribution of dust particles bounded by an infinite plate at $z=0$. A uniform magnetic field $\boldsymbol{B}_{0}$ is acting normal to plate. The fluid as well as the plate is in a state of solid body rotation with constant angular velocity $\Omega$ about the $z$-axis normal to the plate and additionally, non-torsional oscillation of frequency $\omega_{1}$ is imposed on the plate in its own plane. 
An unsteady hydromagnetic dusty fluid flow in a rotating co-ordinate system is governed by the following equations of motion and continuity [6]:

\section{For fluid phase:}

$$
\begin{aligned}
& \frac{\partial \vec{u}}{\partial t}+(\vec{u} \cdot \nabla) \vec{u}+2 \vec{\Omega} \times \vec{u}=-\nabla p_{1}+\frac{1}{\rho}(\vec{J} \times \vec{B})+v \nabla^{2} \vec{u}+\frac{K N}{\rho}(\vec{v}-\vec{u})=0, \\
& \nabla . \vec{u}=0
\end{aligned}
$$

For dust phase:

$$
\begin{aligned}
& m\left[\frac{\partial \vec{v}}{\partial t}+(\vec{v} \cdot \nabla) \vec{v}+2 \vec{\Omega} \times \vec{v}\right]=K(\vec{u}-\vec{v}) \\
& \frac{\partial \lambda}{\partial t}+\nabla \cdot \vec{v}=\mathbf{O}
\end{aligned}
$$

we have the following nomenclature:

$\vec{u}=\left(u_{1}, u_{2}, u_{3}\right)$ and $\vec{v}=\left(v_{1}, v_{2}, v_{3}\right)$ are the velocity of fluid and dust phase respectively, $p$-pressure field including the centrifugal term, $\vec{J}$-electric current density, $\vec{B}$-total magnetic field, $N$-number density of dust particles, $m$-mass of the dust particle, $K$-Stokes-co-efficient of resistance, $\rho$-density, $v$-kinematic viscosity of the fluid, and $\mu_{0}$-magnetic permeability, $t$ - time.

Assuming that the magnetic Reynolds number to be small and neglect the induced magnetic field in comparison with the applied magnetic field. The generalized Ohm's law, in the absence of the electric field is

$$
\vec{J}+\frac{\omega_{e} \tau_{e}}{B_{0}}(\vec{J} \times \vec{B})=\sigma\left[\vec{u} \times \vec{B}+\frac{1}{e n_{e}} \nabla p_{e}\right],
$$

where $\omega_{e}, \tau_{e}, \sigma, e, p_{e}$ and $n_{e}$ are respectively the cyclotron frequency of electrons, electron collision time, electrical conductivity, electron charge, electron pressure and the number density of the electron. The ion-slip and thermoelectric effects are not included in equation (2.5). Further, it is assumed that $\omega_{e} \tau_{e} \approx O(1)$ and $\omega_{e} \tau_{e} \leq 1$, where $\omega_{i}$ and $\tau_{i}$ are cyclotron frequency and collision time for ions respectively.

Assume that the velocity field depends on $z$ and $t$ only, so that

$$
\begin{aligned}
& u(z, t)=\left[u_{1}(z, t), u_{2}(z, t), u_{3}(z, t)\right] \\
& v(z, t)=\left[v_{1}(z, t), v_{2}(z, t), v_{3}(z, t)\right]
\end{aligned}
$$

For the present problem

$$
u_{3}(z, t)=0, \quad v_{3}(z, t)=0 \text { and } \quad N=N_{0} \text { (constant). }
$$

Since it is assumed that, the pressure gradient varying exponentially is impressed on the system for $t>0$, we can write

$$
\frac{1}{\rho} \nabla p_{1}=e^{-\lambda t}
$$

Using the equations (2.6) and (2.7) in the equations of motion (2.1) and (2.3) one can get, 


$$
\frac{\partial u_{1}}{\partial t}-2 \Omega u_{2}=v \frac{\partial^{2} u_{1}}{\partial z^{2}}+\frac{\sigma B^{2}{ }_{0}}{\rho\left(1+m^{2}\right)}\left(m u_{2}-u_{1}\right)-\frac{1}{\tau}\left(u_{1}-v_{1}\right),
$$

$\frac{\partial u_{2}}{\partial t}-2 \Omega u_{1}=v \frac{\partial^{2} u_{2}}{\partial z^{2}}+\frac{\sigma B^{2}}{\rho\left(1+m^{2}\right)}\left(m u_{1}-u_{2}\right)-\frac{1}{\tau}\left(u_{2}-v_{2}\right)$,

$\frac{\partial v_{1}}{\partial t}-2 \Omega v_{2}=-\frac{1}{\tau}\left(u_{1}-v_{1}\right)$

$\frac{\partial v_{2}}{\partial t}-2 \Omega v_{1}=-\frac{1}{\tau}\left(u_{2}-v_{2}\right)$,

where $l=\frac{m N_{0}}{\rho}$ (mass concentration) and $\tau=\frac{m}{k}$ (relaxation time).

Introducing the notation $p=u_{1}+i u_{2}$ and $q=v_{1}+i v_{2}$ in equations (2.10) to (2.13) then they can be written as

$$
\begin{aligned}
& \frac{\partial p}{\partial t}+2 i \Omega p=e^{-\lambda t}+v \frac{\partial^{2} p}{\partial z^{2}}-\frac{\sigma B^{2}{ }_{o}}{\rho\left(1+m^{2}\right)}(1+i m) p+\frac{1}{\rho}(q-p)+\frac{1}{\rho} \frac{\partial p_{1}}{\partial z}, \\
& \frac{\partial q}{\partial t}+2 i \Omega q=\frac{1}{\rho}(p-q) .
\end{aligned}
$$

In view of the imposed oscillations on the plate and consider no-slip boundary condition at the plate and no disturbance at infinity are as

$$
\begin{aligned}
& p(z, t)=p_{0}+\operatorname{Re}\left(A e^{i \omega t}\right) \\
& q(z, t)=q_{0}+\operatorname{Re}\left(B e^{i \omega t}\right) \quad \text { on } \quad z=0, t>0, \\
& p(z, t), q(z, t) \rightarrow 0 \quad \text { as } \quad z \rightarrow \infty, t>0,
\end{aligned}
$$

where $A$ and $B$ are complex constants so that $p(z, t)$ and $q(z, t)$ become real on the plate.

The initial conditions of the problem are taken as $p(z, t)=q(z, t)=0 \quad$ at $t \geq 0$ for all $z$.

\section{Solution Of The Problem}

To make the above system dimensionless, introduce the following non-dimensional variables $z^{\prime}=\frac{z U^{*}}{v}, t^{\prime}=\Omega t, p^{\prime}=\frac{p}{U^{*}}, q^{\prime}=\frac{q}{U^{*}}, C^{\prime}=\frac{v}{U^{* 3}}$ and the non-dimensional parameter $E=\frac{2 \Omega v}{U^{*^{3}}}$ the Eckert number $m=\omega_{e} \tau_{e}$ the Hall parameter $M=\left(\frac{\sigma v B_{0}^{2}{ }_{0}}{\rho U^{*^{2}}}\right)^{\frac{1}{2}}$ the magnetic parameter.

also $\omega=\frac{\omega_{1}}{\Omega}$ is the non-dimensional frequency of oscillation and $\tau^{\prime}=\frac{\tau U^{* 2}}{v}=\frac{m U^{* 2}}{k v}$. 
Using the above non-dimensional variables and parameter in equations (2.14) and (2.15) and the boundary and initial conditions (2.16)-(2.19), can be obtain

$$
\begin{aligned}
& \frac{\partial^{2} p}{\partial z^{2}}-\frac{E}{2} \frac{\partial p}{\partial t}-\left[i E+\frac{M^{2}}{(1-i m)}\right] p+e^{-\lambda_{1} t}-\frac{l}{\tau}(p-q)=0 \\
& \frac{E}{2} \frac{\partial q}{\partial t}+i E q-\frac{1}{\tau}(p-q)=0, \quad z>0, \\
& p=\frac{p_{0}}{U^{*}}+\frac{A e^{i \omega t}}{U^{*}}, \\
& q=\frac{u_{0}}{U^{*}}+\frac{B e^{i \omega t}}{U^{*}} \text { on } z=0, t>0 \\
& p, q \rightarrow 0 \text { as } z \rightarrow \infty, t>0 \\
& p, q=0 \text { at } t \leq 0 \text { for all } z>0 .
\end{aligned}
$$

To solve initial value problem, introduce the Laplace transforms of $p(z, t)$ and $q(z, t)$ respectively as.

$$
\bar{p}(z, s)=\int_{0}^{\infty} e^{-s t} p(z, t) d t \quad \text { and } \quad \bar{q}(z, s)=\int_{0}^{\infty} e^{-s t} q(z, t) d t
$$

On applying the Laplace transforms, to the equations (3.1) and (3.6) one can obtain the solutions for $\bar{p}(z, s)$ and $\bar{q}(z, s)$ as

$$
\begin{aligned}
& \bar{p}=\left[\frac{1}{U^{*}} \frac{p_{0}}{s}+\frac{1}{U^{*}}\left(\frac{A}{s-i \omega}\right)-\frac{1}{k(s+\lambda)}\right] e^{-z \sqrt{k}}+\frac{1}{k(s+\lambda)}, \\
& \bar{q}=\left[\frac{1}{U^{*}} \frac{U_{0}}{s}+\frac{1}{U^{*}}\left(\frac{B}{s-i \omega}\right) e^{z \sqrt{k}}\right]-\left(\frac{2}{E \tau s+2 i E \tau+2}\right)\left[\frac{1}{k(s+\lambda)} e^{-z \sqrt{k}}-\frac{1}{k(s+\lambda)}\right]
\end{aligned}
$$

\subsection{Solutions for Small Times}

The nature of the flow fields $p(z, t)$ and $q(z, t)$ for small times can be determined by the asymptotic behavior of their Laplace transforms for the large value of $|s|$ and are given by

$$
\begin{aligned}
& \bar{p}=\left[\frac{1}{U^{*}} \frac{p_{0}}{s}+\frac{1}{U^{*}}\left(\frac{A}{s-i \omega}\right)-\frac{1}{k(s+\lambda)}\right] e^{-z \sqrt{k}}+\frac{1}{k(s+\lambda)}, \\
& \bar{q}=\left[\frac{1}{U^{*}} \frac{U_{0}}{s}+\frac{1}{U^{*}}\left(\frac{B}{s-i \omega}\right) e^{z \sqrt{k}}\right]-\left(\frac{2}{E \tau s+2 i E \tau+2}\right)\left[\frac{1}{k(s+\lambda)} e^{-z \sqrt{k}}-\frac{1}{k(s+\lambda)}\right]
\end{aligned}
$$

Taking inverse Laplace transforms to equations (3.10) and (3.11) one can get 


$$
\begin{aligned}
& p(z, t)=\frac{A}{2 U^{*}} e^{i \omega t}\left\{e^{z \sqrt{\frac{E i \omega}{2}}} \operatorname{erfc}\left(\frac{z}{2} \sqrt{\frac{E}{2 t}}+\sqrt{i \omega t}\right)+e^{-z \sqrt{\frac{E i \omega}{2}}} \operatorname{erfc}\left(\frac{z}{2} \sqrt{\frac{E}{2 t}}-\sqrt{i \omega t}\right)\right\} \\
& +\frac{p_{0}}{U^{*}} \operatorname{erfc}\left(\frac{z}{2} \sqrt{\frac{E}{2 t}}\right)-\frac{2}{\lambda E} \operatorname{erfc}\left(\frac{z}{2} \sqrt{\frac{E}{2 t}}\right)+\frac{2}{\lambda E}\left(1-e^{\lambda t}\right) \\
& -\frac{2}{\lambda E} e^{-\lambda t}\left\{e^{z \sqrt{\frac{-E i \lambda}{2}}} \operatorname{erfc}\left(\frac{z}{2} \sqrt{\frac{E}{2 t}}+\sqrt{-i \lambda t}\right)+e^{-z \sqrt{\frac{-E i \lambda}{2}}} \operatorname{erfc}\left(\frac{z}{2} \sqrt{\frac{E}{2 t}}-\sqrt{-i \lambda t}\right)\right\} \\
& q(z, t)=\frac{A}{2 U^{*}} e^{i \omega t}\left\{e^{z \sqrt{\frac{E i \omega}{2}}} \operatorname{erfc}\left(\frac{z}{2} \sqrt{\frac{E}{2 t}}+\sqrt{i \omega t}\right)+e^{-z \sqrt{\frac{E i \omega}{2}}} \operatorname{erfc}\left(\frac{z}{2} \sqrt{\frac{E}{2 t}}-\sqrt{i \omega t}\right)\right\} \\
& +\frac{4}{E^{2} \tau}\left[\frac{1}{a_{1} \lambda} \operatorname{erfc}\left(\frac{z}{2} \sqrt{\frac{E}{2 t}}\right)+\frac{1}{a_{1}\left(a_{1}-\lambda\right)} \frac{e^{-a_{1} t}}{2}\left\{\begin{array}{l}
e^{z \sqrt{\frac{a_{1} E}{2}}} \operatorname{erfc}\left(\frac{z}{2} \sqrt{\frac{E}{2 t}}-\sqrt{-a_{1} t}\right) \\
+e^{z \sqrt{\frac{a_{1} E}{2}}} \operatorname{erfc}\left(\frac{z}{2} \sqrt{\frac{E}{2 t}}-\sqrt{-a_{1} t}\right)
\end{array}\right\}\right] \\
& +\frac{4}{E^{2} \tau}\left[\frac{1}{a_{1}\left(a_{1}-\lambda\right)} \frac{e^{\lambda t}}{2}\left\{e^{-z \sqrt{\frac{\lambda E}{2}}} \operatorname{erfc}\left(\frac{z}{2} \sqrt{\frac{E}{2 t}}-\sqrt{-\lambda t}\right)+e^{z \sqrt{\frac{\lambda E}{2}}} \operatorname{erfc}\left(\frac{z}{2} \sqrt{\frac{E}{2 t}}+\sqrt{-\lambda t}\right)\right\}\right] \\
& +\frac{4}{E^{2} \tau}\left\{\frac{-1}{a_{1} \lambda}-\frac{1}{a_{1}\left(\lambda-a_{1}\right)} e^{-a_{1} t}-\frac{1}{\lambda\left(a_{1}-\lambda\right)} e^{-\lambda t}\right\}+\frac{p_{0}}{U^{*}} \operatorname{erfc}\left(\frac{z}{2} \sqrt{\frac{E}{2 t}}\right)
\end{aligned}
$$

From the above solution one can say that immediately after the pulsatile motion is imposed on the plate, an unsteady boundary layer flow builds up in the vicinity of the plate. Further the solution consists of Stokes layer of thickness of order $\sqrt{\frac{v}{\omega}}$ and the Rayleigh layer order $\sqrt{v t}$. Also one can observe that the solution is remains unaffected by the dusty parameter as well as rotation and magnetic term. Similar discussion is true for $q(z, t)$ also.

3.2. Solutions for large Times. Solutions of $p(z, t)$ and $q(z, t)$ can be determined by the asymptotic behavior of their Laplace transforms for the small value of $|s|$ are given by

$$
\begin{aligned}
\bar{p} & =\left[\frac{1}{U^{*}}\left(\frac{A}{s-i \omega}\right)+\frac{p_{0}}{U^{*}} \frac{1}{s}-\frac{1}{k(s+\lambda)}\right] e^{-\sqrt{k x}}+\frac{1}{k(s+\lambda)}, \\
\bar{q} & =\left[\frac{u_{0}}{U^{*}} \frac{1}{s}+\frac{\operatorname{Re}}{U^{*}} \frac{B}{s-i \omega}\right] e^{\sqrt{k z}}-\frac{2}{(E \tau S+2 i E \tau+2) k(s+\lambda)} e^{-\sqrt{k z}} \\
& +\frac{2}{(E \tau S+2 i E \tau+2) k(s+\lambda)}
\end{aligned}
$$

Taking inverse Laplace transforms to equations (3.12) and (3.13) we get 


$$
\begin{aligned}
& p(z, t) \approx \frac{e^{i \omega t}}{2 U^{*}}\left[e^{z \sqrt{\frac{L}{\delta}} \sqrt{\frac{1}{L}+i \omega}} \operatorname{erfc}\left(\frac{z \sqrt{\frac{\gamma L}{\delta}}}{2 \sqrt{t}}+\sqrt{\left(\frac{1}{L}+i \omega\right) t}\right)+e^{-z \sqrt{\frac{L}{\delta}} \sqrt{\frac{1}{L}+i \omega}} \operatorname{erfc}\left(\frac{z \sqrt{\frac{\gamma L}{\delta}}}{2 \sqrt{t}}-\sqrt{\left(\frac{1}{L}+i \omega\right) t}\right)\right] \\
& \left.-\frac{\delta}{\gamma L}\left[\frac{e^{-\lambda t}}{2}\left\{e^{-z \sqrt{\frac{\alpha}{\delta}} \sqrt{\frac{1}{L}-\lambda}} \operatorname{erfc}\left(\frac{z \sqrt{\frac{\gamma L}{\delta}}}{2 \sqrt{t}}-\sqrt{\left(\frac{1}{L}-\lambda\right) t}\right)+e^{z \sqrt{\frac{\zeta}{\delta}} \sqrt{\frac{1}{L}-\lambda}} \operatorname{erfc}\left(\frac{z \sqrt{\frac{\gamma L}{\delta}}}{2 \sqrt{t}}+\sqrt{\left(\frac{1}{L}-\lambda\right)}\right)\right]\right\}\right] \\
& +\frac{p_{0}}{U^{*}}\left\{e^{z \sqrt{\frac{\gamma L}{\delta}}} \operatorname{erfc}\left(\frac{z \sqrt{\frac{\gamma L}{\delta}}}{2 \sqrt{t}}+\sqrt{\frac{t}{L}}\right)+e^{-z \sqrt{\frac{\gamma L}{\delta}}} \operatorname{erfc}\left(\frac{z \sqrt{\frac{\gamma L}{\delta}}}{2 \sqrt{t}}-\sqrt{\frac{t}{L}}\right)\right\}-\frac{\delta}{\gamma L}\left[\frac{1}{\left(\lambda-\frac{1}{L}\right)} e^{-\frac{1}{L}} \operatorname{erfc}\left(\frac{z \sqrt{\frac{\gamma L}{\delta}}}{2 \sqrt{t}}\right)\right] \\
& +\frac{\delta}{\left(\lambda-\frac{1}{L}\right) \gamma L}\left(e^{-\frac{1}{L}}-e^{\lambda t}\right) .
\end{aligned}
$$

$q(z, t) \approx \frac{e^{i \omega t}}{2 U^{*}}\left[e^{z \sqrt{\frac{\gamma L}{\delta}} \sqrt{\frac{1}{L}+i \omega}} \operatorname{erfc}\left(\frac{z \sqrt{\frac{\gamma L}{\delta}}}{2 \sqrt{t}}+\sqrt{\left(\frac{1}{L}+i \omega\right) t}\right)+e^{-z \sqrt{\frac{\gamma L}{\delta}} \sqrt{\frac{1}{L}+i \omega}} \operatorname{erfc}\left(\frac{z \sqrt{\frac{\gamma L}{\delta}}}{2 \sqrt{t}}-\sqrt{\left(\frac{1}{L}+i \omega\right) t}\right)\right]$

$+\frac{p_{0}}{U^{*}}\left\{e^{z \sqrt{\frac{\gamma L}{\delta}}} \operatorname{erfc}\left(\frac{z \sqrt{\frac{\gamma L}{\delta}}}{2 \sqrt{t}}+\sqrt{\frac{t}{L}}\right)+e^{-z \sqrt{\frac{\gamma L}{\delta}}} \operatorname{erfc}\left(\frac{z \sqrt{\frac{\gamma L}{\delta}}}{2 \sqrt{t}}-\sqrt{\frac{t}{L}}\right)\right\}$

$-\frac{2 \delta}{E \tau \nu L}\left[\frac{-1}{\left(\lambda-a_{1}\right)\left(a_{1}-\frac{1}{L}\right)} \frac{e^{-a_{1} t}}{2}\left\{e^{-z \sqrt{\frac{\gamma L}{\delta}} \sqrt{\frac{1}{L}-a_{1}}} \operatorname{erfc}\left(\frac{z \sqrt{\frac{\gamma L}{\delta}}}{2 \sqrt{t}}-\sqrt{\left(\frac{1}{L}-a_{1}\right) t}\right)\right.\right.$

$\left.+e^{z \sqrt{\frac{\gamma L}{\delta} \sqrt{\frac{1}{L}-a_{1}}}} \operatorname{erfc}\left(\frac{z \sqrt{\frac{\gamma L}{\delta}}}{2 \sqrt{t}}+\sqrt{\left(\frac{1}{L}-a_{1}\right) t}\right)\right\}-\frac{1}{\left(a_{1}-\frac{1}{L}\right)\left(\frac{1}{L}-\lambda\right)} e^{-\frac{t}{L}} \operatorname{erfc}\left(\frac{z \sqrt{\frac{\gamma L}{\delta}}}{2 \sqrt{t}}\right)$

$\times\left[\left\{-\frac{1}{\left(\frac{1}{L}-\lambda\right)\left(\lambda-a_{1}\right)} \frac{e^{-\lambda t}}{2}\right\}+\left\{e^{-z \sqrt{\frac{\gamma L}{\delta}} \sqrt{\frac{1}{L}-a_{1}}} \operatorname{erfc}\left(\frac{z \sqrt{\frac{\gamma L}{\delta}}}{2 \sqrt{t}}-\sqrt{\left(\frac{1}{L}-\lambda\right) t}\right)\right\}\right]$

$+e^{z \sqrt{\frac{\gamma L}{\delta}} \sqrt{\frac{1}{L}-a_{1}}} \operatorname{erfc}\left(\frac{z \sqrt{\frac{\gamma L}{\delta}}}{2 \sqrt{t}}+\sqrt{\left(\frac{1}{L}-\lambda\right) t}\right)+\frac{2 \delta}{E \tau \gamma L}\left\{\frac{1}{\left(\frac{1}{L}-a_{1}\right)\left(\lambda-a_{1}\right)} e^{-a_{1} t}+\frac{1}{\left(a_{1}-\frac{1}{L}\right)\left(\lambda-\frac{1}{L}\right)} e^{\frac{t}{L}}\right\}$

$+\frac{2 \delta}{E \tau \gamma L}\left\{\frac{1}{\left(a_{1}-\lambda\right)\left(\frac{1}{L}-\lambda\right)} e^{-\lambda t}\right\}$

Where 
$L=\frac{\beta \delta-\gamma \lambda_{1}}{2 \gamma \delta}, K=\frac{\alpha s^{2}+\beta s+\gamma}{\lambda_{1} s+\delta}, \alpha=\frac{\tau^{2} E^{2}}{4}(1-i m)$

$\beta=\tau^{2} E(1-i m)\left(i E+\frac{1}{2 \tau}\right)+\frac{E}{2} \tau\left(M^{2} \tau-L\right)$,

$\gamma=-E \tau^{2}(1-i m)\left(E-\frac{i}{\tau}\right)+i m^{2} \tau^{2} E+M^{2} \tau+i l E \tau(1-i m)$,

$\lambda_{1}=(1-i m) \tau \frac{E}{2}, \quad \delta=\tau(1-i m)(i E \tau+1)$,

\section{Results and Discussion}

The thickness of the boundary layer changes with the Ekman number, magnetic parameter and Hall current parameter. In fact, the boundary layer thickness increases with increase in Hall current parameter. Similar prediction for Hall current effect is also made by Debnath [3] and R. Tiwari and Kamal Singh [1]. The effect of Ekman number, magnetic parameter and Hall current parameter on fluid and dust velocities are depicted graphically verses $z$.
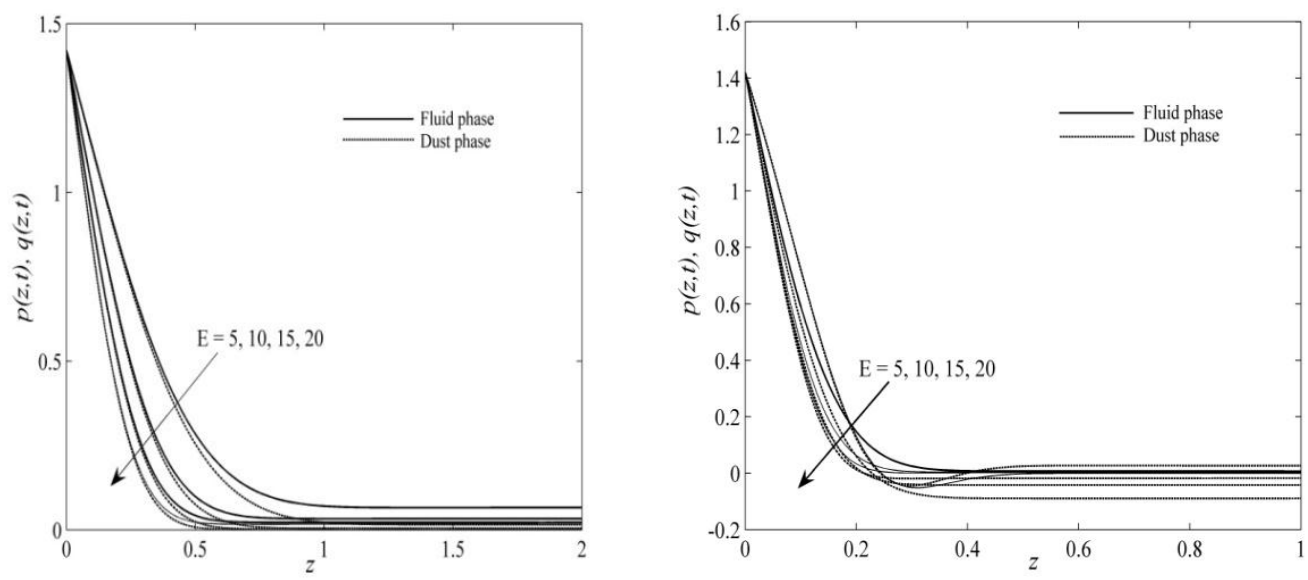

Figure 1: Effect of Ekman number on fluid and dust velocity for small times.
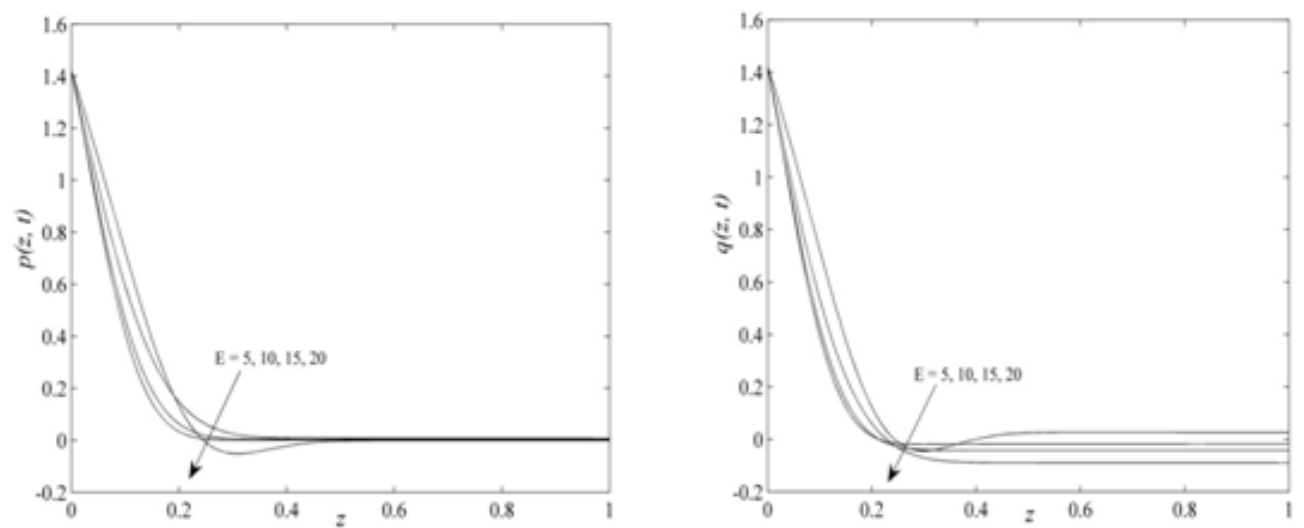

Figure 2: Effect of Elman number on fluid and dust velocity for large times 

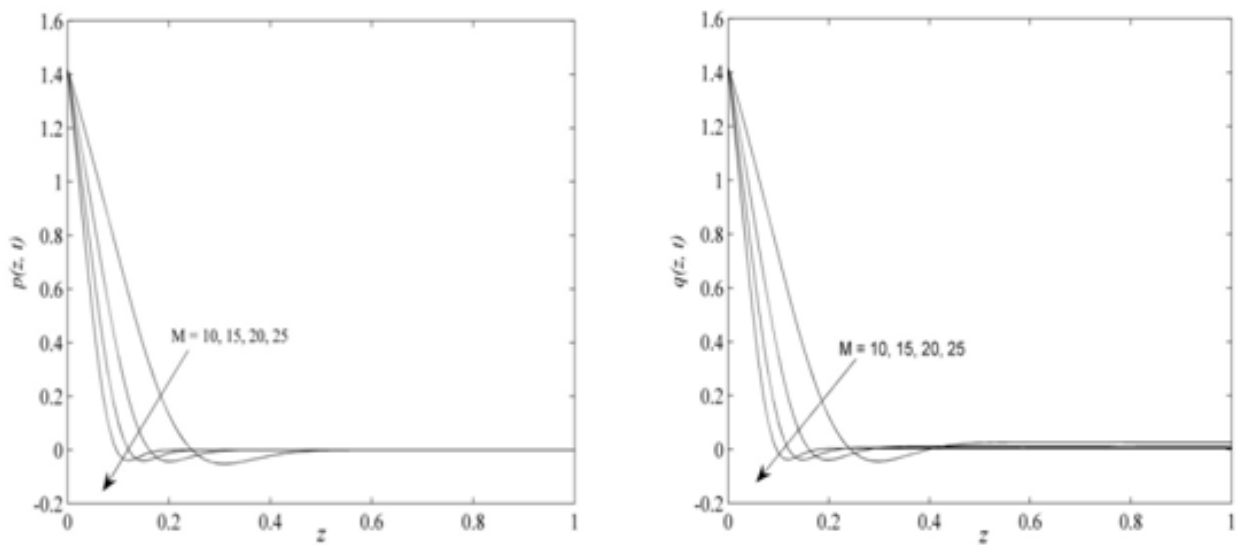

Figure 3: Effect of Magnetic parameter on fluid and dust velocity for large times
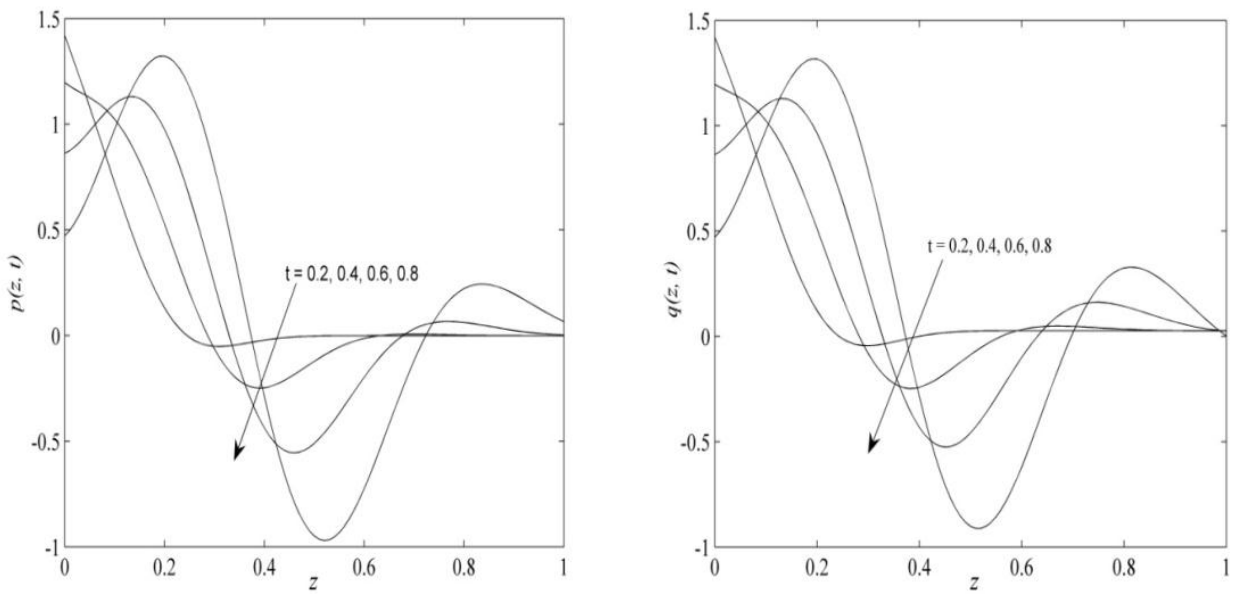

Figure 4: Effect of time on fluid and dust velocity for large times
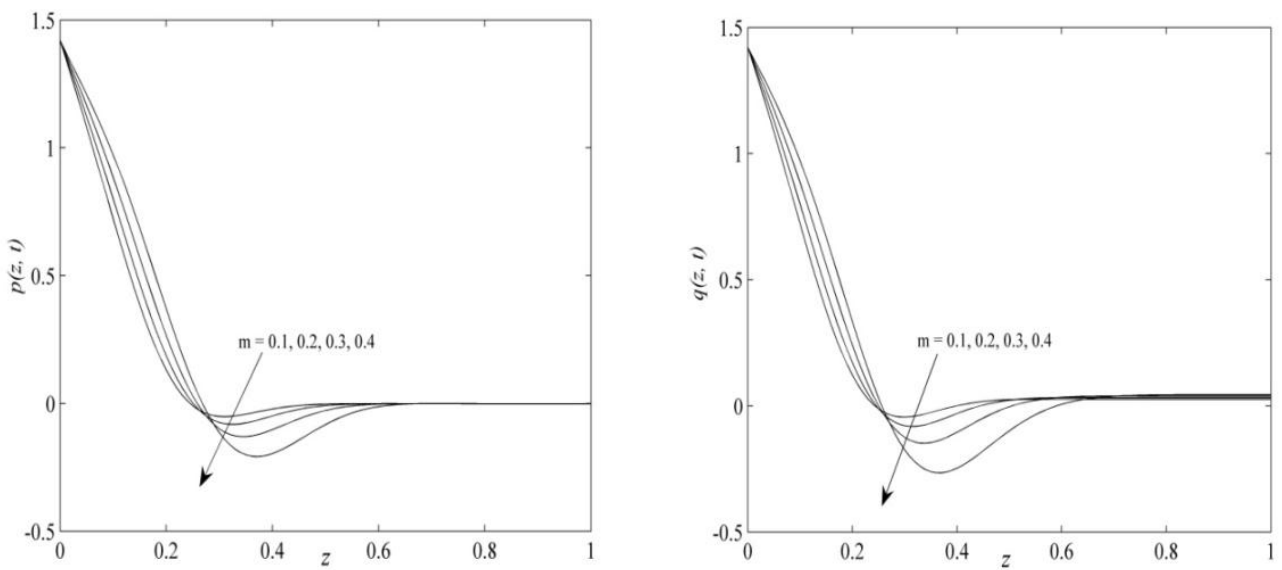

Figure 5: Effect of Hall current parameter on fluid and dust velocity for large times.

Figure 1 and 2 depict the velocity profile for fluid and dust versus $z$, for different values of Ekman number $E$. We infer from these figures that the fluid and dust velocities decrease with the increasing in Ekman number for both small and large times.

The velocity of fluid and dust remains unaffected by magnetic parameter and Hall parameter in small times solution. But in large times both fluid and dust velocity is effected by the magnetic parameter and Hall parameter. 
Here the effect of increasing values of a magnetic parameter $M$ is to decrease the fluid and dust velocity. As $M$ increases, the Lorentz force increases and it leads to enhanced deceleration of the flow, which is as in figure 3.

Figures 4 reveals that the fluid and dust velocity decrease with increase in the time. It is interesting to note that the thickness of boundary decreases with increasing time.

Figures 5 show the effect on velocities of both fluid and dust phase. From these figures one can observe that the fluid and dust velocity decreases with increase in Hall current parameter $m$. i.e one can find the increase in the boundary layer thickness. Similar prediction for Hall current effect is also made by Debnath and others [3].

\section{Conclusions}

In this paper a mathematical analysis has been carried out on momentum characteristics in an incompressible viscous unsteady hydromagnetic boundary layer in rotating dusty fluid in the presence of hall current and time dependent pressure gradient. The governing equations are solved by applying the asymptotic behaviour of Laplace transform treatment. The effect of various physical parameter like Ekman number $E$, magnetic parameter $M$, Hall current parameter $m$ and time $t$ are examined. Some of the important findings of our analysis obtained by the graphical representation are listed below:

- The solution remains unaffected by magnetic parameter and Hall current parameter in small times where as these effect for large times.

- The effect of magnetic parameter $M$ and hall current parameter $m$ is to decrease the fluid and particle velocity.

- The effect of Ekman number $E$ is to decrease the fluid and dust partical velocity.

- The effect of time is to decrease the both fluid and dust velocity.

\section{References}

[1]. R.Tiwari and Kamal Singh, Effect of Hall current on unsteady hydromagnetic boundary layer in rotating dusty fluid, Indian J. Pure appl. Math., 14(1) (1983) 159-165.

[2]. D.R.V. Prasada Rao and D.V.Krishna, Hall effect on unsteady hydromagnetic flow, Indian J. Pure appl. Math., 12(2) (1981) 270276 .

[3]. Debnath.L, Effect of Hall current on unsteady Hydromagnetic flow past a porous plate in a rotating fluid system, ZAMM, 59 (1979) 469.

[4]. Oberhettinger,F. and Badii, Table of Laplace Transforms, Spinger-Verlag,Berlin, (1973).

[5]. K. Kanch, R. N. Jana, Hall effects on unsteady hydromagnetic flow past an eccentrically rotating porous disk in a rotating fluid, Czech. J. Phys. 49(2) (1999) 205-215.

[6]. P.G.Saffman, On the stability of laminar flow of a dusty gas, Journal of Fluid Mechanics, 13(1962) 120-128.

[7]. C.S.Bagewadi and B.J.Gireesha, A study of two dimensional unsteady dusty fluid flow under varying pressure gradient, Tensor, N.S., 64 (2003) 232-240.

[8]. C.S.Bagewadi and B.J.Gireesha, A study of two dimensional steady dusty fluid flow under varying temperature, Int. Journal of Appl. Mech. \& Eng., 94 (2004) 647-653.

[9]. Nakayama.A and Koyama.H, An Analysis for Friction and Heat Transfer Characteristics of Power- Law Non-Newtonian Fluid Flows Past Bodies of Arbitrary Geometrical Configuration, Warme-und Stoffubertragung, 22 (1988), 29-37.

[10]. B.J.Gireesha, C. S. Bagewadi \& B.C.Prasannakumara, Puslsatile flow of an unsetady dusty fluid through channel, Com. Nonlinear Sci. Num. Sim., 14(2009)2103-2110.

[11]. D.H.Michael and D.A.Miller, Plane parallel flow of a dusty gas, Mathematika, 13 (1966) 97-109.

[12]. S.K.Ghosh , O.Anwar Beg and J.Zueco, Hydromagnetic free convection flow with induced magnetic field effect, Maccanica, 45 (2010) 175-185.

[13]. A.C.King, J.Billingham and S.R.Otto, Differential Equations, Cambridge University Press, New York, 2003.

[14]. J.T.C.Liu, Flow induced by an oscillating infinite flat plate in a dusty gas, Phys. Fluids, 9 (1966) 1716-1720. 